\title{
PROJETO VER-SUS: PANORAMA DA ATENÇÃO BÁSICA E DA URGÊNCIA E EMERGÊNCIA NO MUNICÍPIO DE CAMPO GRANDE/MS
}

Resumo

Objetivo: Relatar a vivência de um grupo de acadêmicos de instituições públicas e privadas de diferentes estados do Brasil, dos cursos de serviço social, enfermagem, medicina, psicologia, odontologia e fisioterapia, que foram submetidos a um estágio de vivência, na modalidade de imersão, na realidade do Sistema Único de Saúde do estado de Mato Grosso do Sul através do projeto VER-SUS/MS, nas Unidades de Saúde da atenção básica (UBS e UBSF) e nas Unidades de Urgência e Emergência (UPAs). Método: Trata-se de um estudo descritivo no formato de relato de experiência, das vivências pregressas do VER-SUS/MS. Resultados: A vivência despertou o debate crítico a cerca da saúde pública no Brasil, possibilitando uma visão ampliada do panorama da atenção básica e da urgência e emergência no município de Campo Grande/MS. Conclusões: O VER-SUS trabalha com metodologia ativa na formação de trabalhadores de saúde na perspectiva da interação entre ensino/serviço/comunidade, estimulando a formação de trabalhadores comprometidos eticamente com os princípios e diretrizes do SUS e empenhados na defesa da saúde como direito universal. Período: As vivências ocorreram de 03 a 10 de fevereiro de 2018 em Campo Grande/MS.

Palavras-chave: Saúde. Atenção Básica. Urgência e Emergência. VER-SUS.

\section{VER-SUS PROJECT: PANORAMA OF BASIC ATTENTION AND EMERGENCY AND EMERGENCY IN THE MUNICIPALITY OF CAMPO GRANDE/MS}

\begin{abstract}
Objective: Report the experience of a group of academics from public and private institutions from different states of Brazil, from the courses of social work, nursing, medicine, psychology, dentistry and physiotherapy, who underwent an experience stage in the modality immersion in the reality of the System. Health Unit in the state of Mato Grosso do Sul through the VER - SUS / MS project, in the Primary Care Health Units (UBS and UBSF) and in the Urgency and Emergency Units (UPAs). Method: This is a descriptive study in the format of experience report of previous experiences of VER-SUS / MS. Results: The experience aroused the certain critical debate about public health in Brazil, allowing a broader view of the panorama of primary care and urgency and emergency in the city of Campo Grande / MS. Conclusions: VER-SUS works with an active methodology in the training of health workers from the perspective of the interaction between teaching / service / community, stimulating the formation of workers ethically committed to the principles and guidelines of SUS and committed to the defense of health as a universal right. Period: Experiences occurred from 03 to 10 February 2018 in Campo Grande / MS.

Keywords: Health. Basic Attention. Urgency and emergency. VER-SUS.

\section{PROYECTO VER-SUS: PANORAMA DE LA ATENCIÓN BÁSICA Y DE LA URGENCIA Y EMERGENCIA EN EL MUNICIPIO DE CAMPO GRANDE/MS}

\footnotetext{
Resumen

Reporte la experiencia de un grupo de académicos de instituciones públicas y privadas de diferentes estados de Brasil, de los cursos de trabajo social, enfermería, medicina, psicología, odontología y fisioterapia, que se sometieron a una etapa de experiencia en la inmersión de la modalidad en la realidad del Sistema. Unidad de Salud en el estado de Mato Grosso do Sul a través del proyecto VER - SUS / MS, en las Unidades de Salud de Atención Primaria (UBS y UBSF) y en las Unidades de Urgencia y Emergencia (UPA). Método: La experiencia despertó cierto debate crítico sobre la salud pública en Brasil, permitiendo una visión más amplia del panorama de atención primaria y urgencia y emergencia en la ciudad de Campo Grande / MS. Resultados: La vivencia despertó el debate crítico a cierta de la salud pública en Brasil, posibilitando una visión ampliada del concepto de salud. Conclusiones: El VER-SUS trabaja con metodología activa en la formación de trabajadores de salud en la perspectiva de la interacción entre enseñanza / servicio / comunidad estimulando la formación de trabajadores comprometidos éticamente con los principios y directrices del SUS y comprometidos en la defensa de la salud como derecho universal. Período: Las vivencias ocurrieron de 03 a 10 de febrero de 2018 en Campo Grande / MS. Palavras clave: Salud. Atención Básica. Urgencia y Emergencia. VER-SUS.
} 
Projeto VER-SUS: panorama da atenção básica e da urgência e emergência no município de Campo Grande/MS

\section{INTRODUÇÃO}

O presente estudo apresenta um relado descritivo, sob uma óptica acadêmica, a partir da experiência do estágio de vivência na modalidade de imersão propiciado pelo projeto VERSUS/MS (Vivências e Estágios na Realidade do Sistema Único de Saúde de Mato Grosso do Sul), nas unidades de saúde de atenção básica e de urgência e emergência, no primeiro bimestre do ano de 2018, na cidade de Campo Grande/MS.

A vivência foi realizada por acadêmicos de diferentes cursos (serviço social, enfermagem, medicina, psicologia, odontologia, fisioterapia), vindos de instituições de ensino públicas e privadas, de diversas regiões do país. Ocorreu através da imersão teórico-prático na realidade do Sistema Único de Saúde o processo de ensino-aprendizagem de forma interdisciplinar e multiprofissional.

Segundo Meneses (2019) no contexto do processo de ensino-aprendizagem acadêmico existe a necessidade da inserção de práticas interdisciplinares, para superação do conhecimento fragmentado em disciplinas. A interdisciplinaridade deve ser pensada como um espaço de discussões entre as disciplinas onde seja possível o "encontro entre diferentes saberes, vislumbrando a construção de novos caminhos, através de indivíduos que se dispõem ao desafiante exercício de uma escuta ativa e qualificada na busca de outros possíveis" (MENESES, 2019, p. 347).

O VER-SUS realiza-se sob metodologia de imersão com duração de uma a duas semanas, contando com participação de estudantes de graduação, pós-graduação, residentes, técnicos e membros de movimentos sociais. O projeto recebe inscrições em três modalidades: viventes, facilitadores e comissão organizadora.

Os viventes são os indivíduos (estudantes de graduação, residentes, técnicos e membros de movimentos sociais) que participam da vivência. Os facilitadores são viventes que já participaram do VER-SUS anteriormente e possuem participação e organização em movimentos estudantis e sociais. O facilitador tem como atribuição fomentar as discussões durante a vivência, favorecendo a interlocução com os demais parceiros do projeto, sendo corresponsável pelas tomadas de decisões juntamente com a comissão organizadora, sobre as questões técnicas e operacionais relativas ao processo de vivência (COSTA, 2013).

A Comissão Organizadora é composta preferivelmente por membros de representações estudantis, trabalhadores da saúde e representantes da Participação Popular e dos Conselhos de Saúde, gestores municipais e estaduais de saúde. É responsável por propor e organizar o processo formativo da vivência. 
Projeto VER-SUS: panorama da atenção básica e da urgência e emergência no município de Campo Grande/MS

O VER-SUS pode ser considerado como "ferramenta estratégica na educação em saúde e na formação de novos profissionais agentes da transformação social” (WEBER, 2016, p. 115) necessárias dado ao momento de ataque às políticas sociais públicas e regressão de direitos sociais (PAIM, 2018).

No tocante às vivências, experimentadas no lócus das Unidades de Saúde da atenção básica (UBS e UBSF) e nas Unidades de Urgência e Emergência (UPAs), do município de Campo Grande/MS percebeu-se a precarização do SUS, que se encontra sucateado refletindo diretamente na qualidade da saúde prestada à população.

O retrocesso e o desmonte dos direitos sociais, através dos ataques às políticas públicas, foi reforçada pela crise econômica no Brasil e suas políticas de austeridade fiscal, que podem ser vistas através da Emenda Constitucional 95 (EC-95/2016) que congela despesas primárias, entre elas as da saúde, durante vinte anos (PAIM, 2018).

Na luta pela defesa do SUS, a vivência do projeto VER-SUS vem ao encontro da formação de trabalhadores comprometidos eticamente com a Saúde Pública através de experiência de aprendizado interdisciplinar (BRUFATTO, 2014).

Fomentando a militância na luta pelo direito à saúde no debate ampliado do fortalecimento da cidadania. Propiciando uma formação acadêmico-profissional onde os sujeitos envolvidos refletem "o SUS, suas leis e diretrizes" se "tornando parte do processo por meio da inserção em serviços de saúde cheios de paradigmas" ocorrendo a desmistificação da saúde pública (FETTERMANN, 2018).

Tendo em vista a necessidade da formação de trabalhadores, de forma interdisciplinar, comprometidos eticamente com os princípios e diretrizes do SUS, o VER-SUS promove a interação entre ensino/serviço/comunidade estimulando defesa da saúde como direito universal.

Diante do exposto este estudo tem como objetivo relatar a vivência dos acadêmicos durante o estágio vivencial nas unidades de saúde da atenção básica e nas unidades de urgência e emergência compreendendo a atenção primaria e secundária.

\section{METODOLOGIA}

Trata-se de um estudo descritivo, no formato de relato de experiência, das vivências pregressas do VER-SUS/MS que ocorreram nas cidades de Campo Grande e Três Lagoas no estado de Mato Grosso do Sul, e contemplaram 40 estudantes de 06 cursos diferentes (serviço social, enfermagem, medicina, psicologia, odontologia e fisioterapia). O relato de experiência 
Projeto VER-SUS: panorama da atenção básica e da urgência e emergência no município de Campo Grande/MS

descrito neste trabalho refere-se somente à vivência ocorrida na cidade de Campo Grande com participação de 24 acadêmicos no período de 03 a 10 de fevereiro de 2018.

Durante a vivência os acadêmicos foram acomodados em alojamento conjunto na Universidade Federal de Mato Grosso do Sul, para que dispusessem de maior interação com o grupo e ocorressem momentos de diálogos, discussões e trocas de experiências, propiciando um conhecimento mútuo.

Os 24 acadêmicos foram divididos em 04 grupos (G1, G2, G3 e G4) de 06 pessoas, cada grupo foi formado por 01 facilitador e 05 viventes. Durante o dia, os grupos de acadêmicos foram distribuídos pelas unidades de saúde descritas no "Plano de Vivência" no qual constam as unidades que serão campos de prática do estágio vivencial. Retornando ao alojamento no final da tarde, e realizando no período da noite a discussão em roda de conversa sobre vivência realizada naquele dia.

A vivência em questão foi realizada em 05 unidades de saúde compreendidas entre UBSF (Unidade Básica de Saúde da Família), UBS (Unidade Básica de Saúde) e UPA (Unidade de Pronto Atendimento Unidade Básica de Saúde da Família).

As UBSFs são compostas por equipes da Estratégia de Saúde da Família (ESF) que atendem de forma regionalizada um número determinado de famílias, através de consultas agendadas. Sendo lócus desta vivência a UBSF Los Angeles “Dr. Sebastião Luiz Nogueira” e a UBSF MAPE “Maria Aparecida Pedrossian”. A UBSF Los Angeles atende somente as famílias da área de abrangência, que compreendem os seguintes bairros: Sumatra, Uirapuru, Vespasiano Martins, Jardim Los Angeles, Morada do Sol, Terra Morena e parte do Macaúbas. E a UBSF MAPE atende de forma regionalizada as famílias dos bairros: Residencial Fernando Sabino, Maria Aparecida Pedrossian, Residencial Oiti, Jardim Panorama, Vivendas do Parque e Jardim Samambaia.

A UBS consiste em uma unidade de saúde de porta aberta que atende demandas espontâneas independente da região de moradia do paciente, as consultas são previamente agendadas, ocorrendo se necessário o encaixe para consultas de pacientes não agendados. É palco desta vivência a UBS Dona Neta "Engenheiro Arthur Okama" que assiste todo o território de Campo Grande/MS.

Já a UPA se encarrega dos atendimentos de urgências e emergências de adultos e crianças, realizados por demanda espontânea não necessitando de agendamento prévio. Foram contempladas nesta vivência a UPA Coronel Antonino "Dr. Walfrido Azambuja de Arruda" e a UPA Moreninhas III "Joel Rodrigues Rocha” que atendem a população de qualquer uma das regiões sanitárias do município. 
Projeto VER-SUS: panorama da atenção básica e da urgência e emergência no município de Campo Grande/MS

Os acadêmicos entraram em contato direto com os serviços disponibilizados nas unidades de saúde, interagindo com gestores, trabalhadores da saúde e pacientes atendidos. Participando e observando a rotina, a dinâmica, o fluxo e as especificidades de cada unidade. Após as atividades, desenvolvidas nas unidades de saúde, foi solicitado aos acadêmicos a elaboração de um Portfólio contendo as experiências de aprendizagem, objetivando a reflexão crítica do acadêmico sobre a saúde pública e o fazer profissional na saúde, visando à melhoria das competências profissionais, aprimorando o conhecimento.

Durante a vivência os acadêmicos tiveram a oportunidade de vivenciar na prática toda teoria trabalhada nos cursos universitários, desenvolvendo novas ações em contato direto com a comunidade, com os trabalhadores da saúde e com os gestores. Essa articulação do conhecimento científico, advindo do ensino e da pesquisa, com as necessidades da comunidade, onde se realiza o projeto, transformam o fazer da saúde pública.

A vivência proporcionou aos acadêmicos um processo pedagógico de aprendizagem através da metodologia ativa, valendo-se de rodas de conversas, dinâmicas, teatro, trabalhando a perspectiva da interação entre ensino/serviço/comunidade.

\section{CONTEXTUALIZANDO O PROJETO VER-SUS/BRASIL}

O Projeto VER-SUS/BRASIL é desenvolvido pelo Ministério da Saúde em parceria com a Rede Unida, a Rede Governo Colaborativo em Saúde, a UNE (União Nacional dos Estudantes), o NCOASS (Conselho Nacional de Secretários de Saúde) e com o CONASEMS (Conselho Nacional de Secretarias Municipais de Saúde), ocorrendo em diversas localidades do país, com acadêmicos das mais diversas áreas de formação.

É realizado através de estágios de vivências, que ocorrem por meio do processo de imersão teórica, prática e vivencial dentro do sistema de saúde da região, onde o projeto está acontecendo, sendo estruturado a partir de seminários, oficinas e palestras e rodas de conversas. A vivência permite a aproximação dos acadêmicos ao cotidiano do Sistema Único de Saúde permitindo assim uma análise dos desafios e potencialidades que este sistema oferece (BRUFATTO, 2014).

A vivência com acadêmicos de cursos de diversas áreas propicia uma grande troca de experiências, permitindo conhecer diferentes pontos de vista sobre um mesmo assunto, possibilitando conhecer a realidade de outras regiões do Brasil. De acordo com Ramos (2019, p. 4691) "as vivências propiciam inúmeros momentos que a graduação não traz, como a interdisciplinaridade, a importância do trabalho em equipe", pode-se dizer que as vivências 
Projeto VER-SUS: panorama da atenção básica e da urgência e emergência no município de Campo Grande/MS

estabelecem novas percepções sobre a própria formação acadêmica e profissional, exercitando o trabalho multiprofissional e interdisciplinar.

O projeto possibilita percorrer por diversos serviços, instituições e organizações que trabalham sob a égide do Sistema Único de Saúde, impactando assim no próprio serviço de saúde que recebe os acadêmicos, permitindo acompanhar os processos de gestão, participação social no exercício do controle social e educação na saúde (BRUFATTO, 2014).

Carvalho e Silva apontam (2016, p. 459) que o VER-SUS possibilita uma "permissibilidade de observação, discussão e contestação" tornando-se uma ferramenta para a desconstrução de ideias que foram compenetradas na sociedade em relação ao SUS desde a sua gênese, "por grupos interessados em desmoralizar o sistema por meio de desfinanciamento, sucateamento e recentralização", reformulando e reafirmando a percepção dos acadêmicos sobre o SUS.

\section{PANORAMA DA ATENÇÃO BÁSICA NO MUNICÍPIO DE CAMPO GRANDE}

Durante o período de estágio de vivências nas referidas UBSFs e UBS, unidades estas que compõem a atenção básica, pode-se perceber os avanços que a PNAB (Política Nacional de Atenção Básica) alcançou e o que ainda falta para ser alcançado.

Um dos avanços observados refere-se à gradativa transformação das UBSs em UBSFs, pois a atenção básica tem a Saúde da Família como estratégia prioritária para a organização do fluxo dos serviços nas redes de saúde. Esse modelo vida romper com a fragmentação do modelo tradicional "biomédico", voltado para a doença com centralidade no médico. Propondo o modelo de atenção biopsicossocial, voltado para uma visão ampla de saúde, considerando os determinantes e condicionantes da saúde que interferem diretamente no processo saúde-doença.

Em contra-partida um dos problemas observado refere-se ao financiamento das ações da atenção básica, que de acordo com a PNAB (BRASIL, 2017b. p. 29) “deve ser tripartite e com detalhamento apresentado pelo Plano Municipal de Saúde garantido nos instrumentos conforme especificado no Plano Nacional, Estadual e Municipal de gestão do SUS”. Os recursos disponibilizados para a atenção básica, ainda permanecem escassos, sendo necessário que sejam ampliados considerando as desigualdades regionais e as necessidades de cada região sanitária de saúde de Campo Grande/MS.

Outro ponto que chama a atenção diz respeito à Emenda Constitucional 95 (EC95/2016), que impacta diretamente no financiamento da atenção básica, não permitindo o ajustamento de "valores transferidos pelo Ministério da Saúde para estados e municípios", 
Projeto VER-SUS: panorama da atenção básica e da urgência e emergência no município de Campo Grande/MS

dificultando "propostas de redistribuição de recursos" que permitiriam "suprir as necessidades de financiamento dos entes subnacionais com piores condições financeiras”, e impossibilitando a elaboração "de políticas de investimento voltadas para a superação das desigualdades regionais em saúde”. Esta redução do gasto público, durante 20 anos, presente na EC-95/2016 fará com que despesas não acompanhem "o crescimento da renda e da população" acarretando um subfinanciamento da atenção básica (CASTRO; LIMA; MACHADO, 2018, p. 7).

A atenção básica também chamada de atenção primária promove o atendimento inicial dos usuários, objetivando a promoção, proteção e prevenção de doenças. Direciona os casos mais graves para níveis de maior complexidade. A Portaria $n^{\circ} 2.436$, de 21 de setembro de 2017, em seu art. $2^{\circ}$, traz o conceito de atenção básica como sendo:

[...] o conjunto de ações de saúde individuais, familiares e coletivas que envolvem promoção, prevenção, proteção, diagnóstico, tratamento, reabilitação, redução de danos, cuidados paliativos e vigilância em saúde, desenvolvida por meio de práticas de cuidado integrado e gestão qualificada, realizada com equipe multiprofissional e dirigida à população em território definido, sobre as quais as equipes assumem responsabilidade sanitária (BRASIL, 2017b, p. 1)

A Atenção Básica funciona como porta de entrada para os serviços de saúde, sendo um meio de acesso aos Serviços de Especialidades, porém nota-se um aumento no ingresso ao sistema de saúde por meio da atenção especializada (ambulatorial especializada; hospitalar e urgência e emergência). Isso ocorre devido às dificuldades de acesso à atenção básica, levando ao agravamento do caso e consequentemente à procura e ao encaminhamento à atenção especializada.

\footnotetext{
Conforme o Ministério da Saúde, cada 10 vezes que alguém procura um serviço de saúde, em 8 delas o problema pode ser resolvido na Atenção Básica. Vacinação, pré-natal, diagnósticos clínicos, acompanhamento de doenças crônicas, saúde mental, tratamento de doenças como diarreia e amidalite, atendimento de pequenas urgências, cuidados à saúde da mulher, da criança, do adulto e do idoso, entrega gratuita de medicamentos, entre tantos outros são procedimentos realizados na Unidade Básica de Saúde (UBS), ou posto de saúde, cujo papel é atender a comunidade.(VENTURINI, 2017, online)
}

Durante a vivência percebeu-se que a PNH (Política Nacional de Humanização) possui uma variação maior de implementação, estando mais presentes em algumas unidades e apresentando-se timidamente em outras.

A PNH foi editada em 2003, buscando pôr em prática os princípios e diretrizes do SUS no cotidiano de cada um dos serviços de saúde, a fim de promover mudanças no modo de gerir e cuidar da saúde, propiciando a comunicação entre gestores, trabalhadores e usuários para construir uma saúde com mais qualidade, buscando a autonomia e a corresponsabilidade dos 
Projeto VER-SUS: panorama da atenção básica e da urgência e emergência no município de Campo Grande/MS

profissionais de saúde em seu trabalho e dos usuários no cuidado com a própria saúde, por meio de práticas humanizadas (BRASIL, 2004).

\begin{abstract}
A Humanização, como uma política transversal, supõe necessariamente que sejam ultrapassadas as fronteiras, muitas vezes rígidas, dos diferentes núcleos de saber/poder que se ocupam da produção da saúde (...). Como política, a Humanização deve, portanto, traduzir princípios e modos de operar no conjunto das relações entre profissionais e usuários, entre os diferentes profissionais, entre as diversas unidades e serviços de saúde e entre as instâncias que constituem o SUS (...) (BRASIL, 2004, p. 07).
\end{abstract}

Algumas das unidades visitadas atendendo as diretrizes gerais para a implementação da PNH nos diferentes níveis de atenção, adequaram os serviços ao ambiente e a cultura local, promovendo uma ambiência acolhedora e confortável.

Em específico uma das unidades visitadas, destacou-se pela ambiência proporcionada aos usuários. A unidade encontrava-se localizada em um prédio muito antigo, de aparência pouco acolhedora. Pensando na melhora do ambiente a equipe de saúde se reuniu com a comunidade e por meio de uma ação que contou com trabalhos voluntários de funcionários e usuários, pintou a unidade de tal maneira que o ambiente ficasse aconchegante, com uma estética melhor, onde os pacientes se sentissem confortáveis e acolhidos.

Foram feitas várias pinturas de boas vindas, em cores vibrantes espalhadas pelas paredes do prédio, desenhos de personagem infantis trazendo alegria para as crianças, deixando o ambiente da unidade de saúde mais agradável. Ilustrações coloridas na sala de vacina que ajudaram a diminuir a tensão que o ambiente gera nas crianças e nos pais.

A equipe de saúde também disponibilizou um espaço com algumas mesas e cadeiras infantis. Colocaram sobre elas algumas revistas para que as crianças pudessem ler, enquanto aguardavam pelas consultas. Pequenos gestos que atendendo à solicitação da ambiência posta pela $\mathrm{PNH}$, transformaram a maneira de se trabalhar a saúde.

O termo ambiência refere-se à atitude de criar espaços saudáveis, acolhedores e confortáveis. Respeitando a privacidade e propiciando a mudança no processo de trabalho, podendo ser realizada a partir da construção de projeto arquitetônico, das reformas e do uso dos espaços de acordo com as necessidades de usuários e trabalhadores, respeitando sempre a acessibilidade (BRASIL, 2013).

O acolhimento também vem sendo trabalhado por algumas unidades de saúde visitadas. Em algumas unidades ele é mais forte e presente e em outras ainda encontra-se em processo de maturação.

Pode-se definir o acolhimento como sendo uma escuta qualificada oferecida pelos trabalhadores às necessidades do usuário, pois, o ato de acolher reconhece "o que o outro traz 
Projeto VER-SUS: panorama da atenção básica e da urgência e emergência no município de Campo Grande/MS

como legítima e singular necessidade de saúde", garantindo o acesso oportuno desses usuários aos serviços adequados às suas demandas. (BRASIL, 2013, p.8)

O acolhimento deve comparecer e sustentar a relação entre equipes/serviços e usuários/ populações. Como valor das práticas de saúde, o acolhimento é construído de forma coletiva, a partir da análise dos processos de trabalho e tem como objetivo a construção de relações de confiança, compromisso e vínculo entre as equipes/serviços, trabalhador/equipes e usuário com sua rede socioafetiva (BRASIL, 2013, p. 7)

Algumas das UBSFs e UBS já instituíram o serviço de acolhimento dentro unidade, sendo este serviço realizado através da figura do "agente acolhedor". Este "agente acolhedor" é um dos profissionais da própria unidade, que posicionado na porta de entrada, realiza o acolhimento do usuário que adentra o serviço de saúde, prestando as informações necessárias, fazendo a referência e a contrarreferência, encaminhando e direcionando o fluxo.

Este serviço tem melhorado consideravelmente a dinâmica das unidades, diminuindo filas e esperas desnecessárias, pois direciona o paciente ao serviço correto facilitando o seu atendimento. Em algumas unidades o agente acolhedor é sempre um agente de saúde comunitário (ACS), em outras é qualquer um dos profissionais da unidade, que estiverem na escala do acolhimento, seja ele um enfermeiro, técnico de enfermagem, assistente social ou auxiliar administrativo.

Apesar de grandes avanços em relação à PNAB (Política Nacional da Atenção Básica) e à PNH (Política Nacional de Humanização) alguns problemas ainda permanecem latentes, como a falta de recursos humanos e recursos materiais.

A maioria das unidades, onde ocorreram as vivências, estão prestando o serviço com um número reduzido de funcionários, em algumas faltam médicos, enfermeiros, farmacêuticos, assistentes sociais. Os materiais são escassos e as equipes de saúde trabalham no limite, o que muitas vezes acarreta um atendimento de baixa qualidade e uma precária assistência à saúde.

\section{PANORAMA DA URGÊNCIA E EMERGÊNCIA NO MUNICÍPIO DE CAMPO GRANDE-MS}

Campo Grande é uma capital com cerca de 874.210 moradores segundo estimativa do Instituto Brasileiro de Geografia e Estatística (IBGE) e opera com 06 UPAs (Unidades de Pronto Atendimento) e 03 CRS (Centros Regionais de Saúde)com atendimento 24 horas por dia (IBGE, 2017).

O que deixa claro a tendência do emprego do modelo biomédico, organizado através de práticas de saúde com foco nas queixas dos indivíduos que procuram os serviços de saúde, 
Projeto VER-SUS: panorama da atenção básica e da urgência e emergência no município de Campo Grande/MS

atuando diretamente na identificação de sinais e sintomas, tratando apenas a doença, e não atuando na promoção da saúde (FERTONANI, et al., 2014).

De acordo com a Portaria no 10, de 03 de janeiro de 2017(BRASIL, 2017a), que "redefine as diretrizes de modelo assistencial e financiamento de UPA 24h de Pronto Atendimento como componente da Rede de Atenção às Urgências, no âmbito do Sistema Único de Saúde" e considerando a variação de "acordo com a realidade loco regional" que pode gerar o "aumento de demanda por doenças respiratórias" dentre outras que possam impactar a clínica pediátrica e a clínica de adultos/idosos, tem-se o seguinte quadro de proporção de UPAs por habitantes:

QUADRO 1- Proporção de UPAs por habitantes

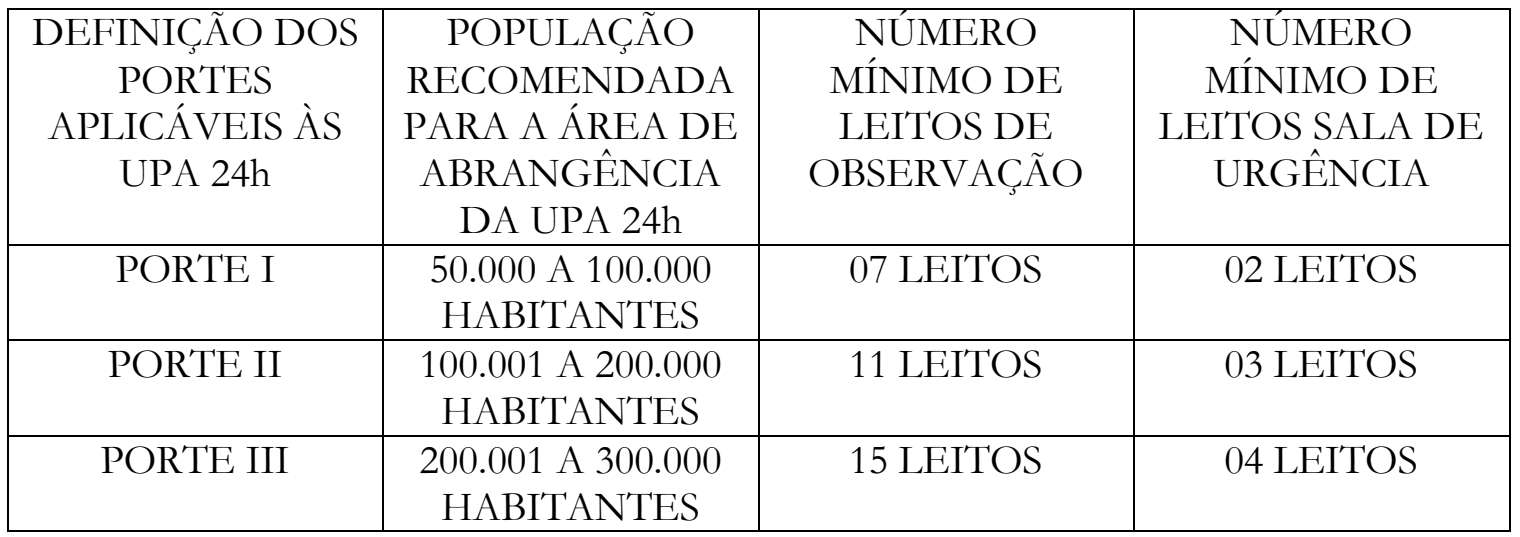

Fonte: Portaria no 10, de 03 de janeiro de 2017. Disponível em:

http://bvsms.saude.gov.br/bvs/saudelegis/gm/2017/prt0010_03_01_2017.html. Acesso em: 11 ago. 2019

A tabela evidência a tendência preponderante no município de Campo Grande da adoção do modelo "medicocêntrico" voltado para o tratamento da doença e não da prevenção, modelo este configurado a partir do modelo "hospitalocêntrico" onde a unidade de saúde funciona como centro do sistema de saúde, e o médico seria o único a tomar as decisões, centrado no modelo apenas curativo e não preventivo (FARIA, et al., 2010).

O modelo "medicocêntrico" é contrario a perspectiva do modelo biopsicossocial baseado no atendimento ambulatorial e comunitário, este modelo considerada que os fatores biológicos, psicológicos e sociais têm importante papel no processo saúde-doença (MARCO, 2015).

O modelo biopsicossocial traz uma proposta de trabalho multidisciplinar e interdisciplinar, pressupondo ações integradas com vistas à prevenção e promoção da saúde em vez do tratamento, entendendo o processo de adoecimento dos sujeitos em sua dimensão física, psicológica e social.

Para Marco (2015) a perspectiva do modelo biopsicossocial tem-se evidenciado progressivamente proporcionando: 
Projeto VER-SUS: panorama da atenção básica e da urgência e emergência no município de Campo Grande/MS

[...] uma visão integral do ser e do adoecer que compreende as dimensões física, psicológica e social,quando incorporada ao modelo de formação do médico coloca a necessidade de que o profissional, além do aprendizado e evolução das habilidades técnico instrumentais, evolua também as capacidades relacionais que permitem o estabelecimento de um vinculo adequado e uma comunicação efetiva (MARCO, 2015, p.64).

O grande investimento na construção e ampliação das unidades de saúde de pronto atendimento que atendem urgências e emergência, somadas ao baixo investimento na atenção básica onde se realiza o atendimento ambulatorial, provoca a superlotação das UPAs de Campo Grande/MS.

Notou-se durante o período de vivência, que o paciente com demanda de ambulatório não conseguindo atendimento nas UBSs e UBSFs, procura atendimento nas UPAs e por vezes acaba por não sanar seu problema de saúde, tendo em vista que o atendimento da UPA é emergencial.

Outro ponto observado na vivência, no que tange à forma de atendimento das UPAs, diz respeito à relação "Usuário X Atendimento de Saúde". As UPAs têm uma forma de atendimento diferenciada das UBSF e UBS, o que por vezes torna a relação com o usuário conflituosa.

Nas unidades básicas as consultas são agendadas anteriormente, sendo o paciente atendido por ordem de chegada. Na UPA o atendimento se dá pela classificação de risco, através do Protocolo de Manchester, ou seja, o paciente mais grave será atendido primeiro independentemente da ordem de chegada. Como não existem ações de Educação em Saúde voltadas para a população a maioria das pessoas desconhecem as diferenças entre o funcionamento destas unidades de saúde. Isso tende a acarretar grandes discussões dos pacientes com os profissionais da saúde, pois aqueles se sentem prejudicados quando um paciente que chegou mais tarde é atendido primeiro do que o paciente que já está há mais tempo aguardando.

O protocolo de Manchester foi realizado pela primeira vez na cidade de Manchester em 1997, sendo baseado em categorias de sinais e sintomas. Contendo 52 fluxogramas que serão selecionados a partir da situação/queixa apresentada pelo paciente, consistindo em um sistema de triagem baseado em cinco cores: vermelho, laranja, amarelo, verde e azul. Sendo o vermelho utilizado para representar os casos de maior gravidade, e azul os casos de menor gravidade (FERNANDES, 2016).

Segundo art. $1^{\circ}$ da Resolução COFEN 311/2007, o enfermeiro é o único profissional da equipe que pode atuar no processo de classificação de risco e priorização da assistência à saúde (FERNANDES, 2016). 
Projeto VER-SUS: panorama da atenção básica e da urgência e emergência no município de Campo Grande/MS

Quadro 2- Representação do Protocolo de Manchester

\begin{tabular}{|l|l|l|l|}
\hline & Prioridade & Cor & Tempo \\
\hline 1 & Emergente & Vermelho & 0 minutos \\
\hline 2 & Muito Urgente & Laranja & 10 minutos \\
\hline 3 & Urgente & Amarelo & 60 minutos \\
\hline 4 & Pouco Urgente & Verde & 120 minutos \\
\hline
\end{tabular}

Fonte: Elaborado através dos dados da pesquisa do autor

Um dos pontos críticos no atendimento das UPAs em Campo Grande refere-se à superlotação e à demora no atendimento. Analisando a situação pode-se perceber que a maioria dos atendimentos das UPAs são triados como azul, ou seja, não são demandas para a urgência e emergência e sim demanda para a unidade básica. São casos a serem resolvidos pelas equipes de saúde dos ambulatórios, e seguindo o Protocolo de Manchester um paciente triado como azul (Não Urgente) teria um prazo de espera de até 4 horas (240 minutos).

Tendo em vista a situação vivenciada através do projeto VER-SUS, formulou-se a seguinte indagação: por que que existem tantas demandas de ambulatório sendo atendidas pela Urgência e Emergência?

Para responder tal questionamento foram levantados os seguintes pontos:

- A falta de médicos na Unidade Básica de Saúde;

- Consultas agendadas com um tempo de espera muito longo (ex: consultas agendadas quinzenalmente ou mensalmente);

- O desconhecimento sobre o que seria demanda para a atenção básica e o que seria demanda para a urgência e emergência;

- O horário de atendimento na UBS e UBSF incompatível ao horário de trabalho do paciente, que encontra um caminho alternativo através do atendimento por meio da UPA;

- O fato de conseguir uma "consulta" no mesmo dia nas UPA, pois a UBS e UBSF realizam consultas por meio de agendamento, então o paciente tem a impressão que na UPA a "consulta" seria mais rápida;

- O paciente não faz o acompanhamento regularmente na UBS por baixa adesão ao tratamento, por barreiras institucionais, barreiras socioculturais e recorre ao UPA para troca de receita de remédios de uso continuo. 
Projeto VER-SUS: panorama da atenção básica e da urgência e emergência no município de Campo Grande/MS

\section{CONSIDERAÇÕES FINAIS}

Todo o processo de ensino-aprendizagem devolvido durante o período de vivência do projeto VER-SUS, na modalidade de imersão nas UBSFs, UBS e UPAs, estimulou o pensamento crítico e reflexivo sobre Atenção Básica e Urgência e Emergência no município de Campo Grande/MS. Possibilitando a interação com diferentes profissões da área da saúde, proporcionando o contato direto com o SUS da realidade, que por vezes difere do SUS da teoria acadêmica.

O VER-SUS leva o estudante a pensar como a prática do fazer profissional pode melhorar a saúde, pois o SUS é construído diariamente por cada trabalhador, usuário e gestor que dele participa ativamente.

O projeto possibilita uma pequena amostra para o acadêmico, sobre como será a prática do profissional recém-formado, quando ocorrer sua inserção no mercado de trabalho, na perspectiva do SUS, e nele incluído suas dificuldades, possibilidades e potencialidades.

A vivência na realidade do SUS constitui-se de um importante dispositivo que permite aos acadêmicos partilharem de um novo espaço de aprendizagem. Contribuindo para uma formação técnica, científica e política abrangendo todos os atores envolvidos.

Trabalhando uma perspectiva multiprofissional, interdisciplinar e intersetorial, reacendendo em cada acadêmico os ideais da reforma sanitária, da luta pelo direito à saúde de forma integral e gratuita, e a defesa do SUS.

\section{REFERÊNCIAS}

BRASIL. Ministério da Saúde. Política Nacional de Humanização. Brasília-DF: Humanizasus, 2013. Disponível em:

http://bvsms.saude.gov.br/bvs/publicacoes/politica_nacional_humanizacao_pnh_folheto.pdf. Acesso em: 24 fev. 2018

BRASIL. Ministério da Saúde. Portaria no 10, de 03 de janeiro de 2017a. Redefine as diretrizes de modelo assistencial e financiamento de UPA 24h de Pronto Atendimento como Componente da Rede de Atenção às Urgências, no âmbito do Sistema Único de Saúde. Disponível em: http://bvsms.saude.gov.br/bvs/saudelegis/gm/2017/prt0010_03_01_2017.html Acesso em: 20 fev. 2018

BRASIL. Ministério da Saúde. Portaria n ${ }^{\circ}$ 2.436, de 21 de setembro de 2017b. Aprova a Política Nacional de Atenção Básica, estabelecendo a revisão de diretrizes para a organização da Atenção Básica, no âmbito do Sistema Único de Saúde (SUS). Disponível em:

http://bvsms.saude.gov.br/bvs/saudelegis/gm/2017/prt2436_22_09_2017.html Acesso em: 24 fev. 2018 
Projeto VER-SUS: panorama da atenção básica e da urgência e emergência no município de Campo Grande/MS

BRASIL. Ministério da Saúde. Secretaria-Executiva. Núcleo Técnico da Política Nacional de Humanização. Humaniza SUS: Política Nacional de Humanização: a humanização como eixo norteador das práticas de atenção e gestão em todas as instâncias do SUS / Ministério da Saúde, Secretaria-Executiva, Núcleo Técnico da Política Nacional de Humanização. - Brasília: Ministério da Saúde, 2004.

BRUFATO, Fabiano. VER-SUS Brasil. Rede Governo Colaborativo em Saúde. 2014. Disponível em: http://www.redegovernocolaborativo.org.br/pastas-antigas/projetos/ver-susbrasil. Acesso: 20 fev. 2018

CARVALHO, Vanessa Lôbo de; SILVA, Carilane Barreto da. Paradigms of operation of the unified health system: perception of a participant of VER SUS. Journal of Nursing UFPE on line, [S.l.], v. 11, n. 1, p. 458-461, dez. 2016. ISSN 1981-8963.Dispinível em : https://periodicos.ufpe.br/revistas/revistaenfermagem/article/view/13577/16378 . Acesso em: 10 ago. 2019.

CASTRO, Ana Luisa Barros; LIMA, Luciana Dias; MACHADO, Cristiani Vieira.

Financiamento da atenção básica no SUS. In: Seminários Preparatórios Abrascão 2018: "De Alma Ata à Estratégia Saúde da Família: 30 anos de APS no SUS - avanços, desafios e ameaças". Rio de Janeiro: ABRASCO, 2018.

COSTA, Drean Falcão. et al. O papel do facilitador no projeto Vivências e Estágios na Realidade do Sistema Único de Saúde (VERSUS) Unisc: um relato de experiência. In: IV Salão de Ensino e de Extensão, 2013, Santa Cruz do Sul. Anais do IV Salão de Ensino e de Extensão. Santa Cruz do Sul: EDUNISC, 2013.

FARIA, Horácio Pereira. et al. Modelo assistencial e atenção básica à saúde. 2 ed. Belo Horizonte: Editora Coopmed,2010. Disponível em:

https://www.nescon.medicina.ufmg.br/biblioteca/imagem/1792.pdf . Acesso em: 24 fev. 2018

FERNANDES, Vinicius. A Classificação de Risco nos Serviços de Urgência e Emergência. 2016. Disponível em: http://www.enfermeiroaprendiz.com.br/classificacao-de-risco-nosservicos-de-urgencia-e-emergencia/. Acesso em: 21 fev.2018

FERTONANI, Hosanna Pattrig et al. Modelo assistencial em saúde: conceitos e desafios para a atenção básica brasileira. Ciência e Saúde Coletiva.2014. Disponível em: http://www.scielo.br/pdf/csc/v20n6/1413-8123-csc-20-06-1869.pdf. Acesso em: 20 fev. 2018

FETTERMANN, Fernanda Almeida et al. VER-SUS project: Influences on the training and performance of nurses. Rev. Bras. Enferm., Brasília , v. 71, n. 6, p. 2922-2929, Dez. 2018. Disponível em: http://www.scielo.br/scielo.php?script=sci_arttext\&pid=S003471672018000602922\&lng=en\&nrm=iso . Acesso em: 10 Ago. 2019.

IBGE. O Brasil em Síntese. 2017. Disponível em: https://cidades.ibge.gov.br/brasil/ms/campo-grande/panorama. Acesso em: 24 fev. 2018

MARCO, Mario Alfredo. Do modelo biomédico ao modelo biopsicossocial: um projeto de educação permanente. Rev. bras. educ. med., Rio de Janeiro, v. 30, n. 1, p. 60-72, Abr. 2006. Disponível em: http://www.scielo.br/scielo.php?script=sci_arttext\&pid=S010055022006000100010\&lng=en\&nrm=iso. Acesso em: 11 Ago. 2019. 
Projeto VER-SUS: panorama da atenção básica e da urgência e emergência no município de Campo Grande/MS

MENESES, Aérica de Figueiredo Pereira. Seminários interdisciplinares como instrumento de articulação de saberes: um relato de experiência. Revista Eletrônica de Educação, v. 13, n. 1, p. 236-349, jan./abr. 2019.

PAIM, J. S. Sistema Único de Saúde (SUS) aos 30 anos - Thirty years of the Unifed Health System (SUS). Ciência \& Saúde Coletiva, v. 23, n. 6, p. 1723-1728, 2018.

RAMOS, Tierle Kosloski et al. Experiences and stages in the unified health system reality - lrsuhs: related experience. Journal of Nursing UFPE on line, [S.l.], v. 10, n. 12, p. 4687-4691, out. 2016. ISSN 1981-8963. Disponível em:

https://periodicos.ufpe.br/revistas/revistaenfermagem/article/view/11539/13445. Acesso em: 10 ago. 2019.

VENTURINI, Dantielle. Cuiabá realiza apenas $38 \%$ das consultas recomendadas. Gazeta Digital. 2017. Disponível em: http://www.gazetadigital.com.br/editorias/cidades/cuiaba-realizaapenas-38-das-consultas-recomendadas/516608. Acesso em: 22 fev. 2018

WEBER, Aldair et al. Vivências e estágios na realidade do SUS (VER-SUS) e a formação profissional em saúde: relato de experiência.Extensio: Revista Eletrônica de Extensão, Florianópolis, v. 13, n. 23, p. 112-122, set. 2016. ISSN 1807-0221. Disponível em:

https://periodicos.ufsc.br/index.php/extensio/article/view/1807-0221.2016v13n23p112/32681. Acesso em: 10 ago. 2019.

Recebido em: 22/10/2018

Aceito em: 16/08/2019 